\title{
Morpho-Active Materials: Fabricating auxetic structures with bioinspired behavior
}

XXIV International Conference of the Iberoamerican Society

of Digital Graphics Medellin | Colombia
Heidi Jalkh

Universidad de Buenos Aires (UBA, FADU) y Humboldt-Universität zu Berlin (HU) | Argentina | heidijalkh@gmail.com

\begin{abstract}
This practice-led research lies at the intersection of design, craft, materials science, and biology. Inspired by the responsive mechanism of plant's biological actuators, and Nature's outstanding capacity of attaining maximal performances while using minimum resources. This thesis explores how to achieve a higher level of integration between the generation of form and behavior with its materialization and fabrication.

This research proposes to endow a conventional laminar elastic material with unconventional behavior. Taking as inspiration plants biological actuators, which allows them to sense and adapt according to different environmental stimuli. We explored, developed, and fabricated a range of cellular structures (and in particular auxetics) that have out of the plane shape morphing capabilities, displaying a distinctive behavior in response to a design pattern (spatial cell arrangement) and an actuating force.
\end{abstract}

The final design is a material/geometry-based actuator with reversible behavior, an active material with integrated tunable and responsive capacity which provides the capabilities to sense, adapt and respond to external stimuli within the structure of the material.

Keywords: Bioinspired; Auxetic Materials; Shape-shifting; Active matter; Soft matter.

\section{INTRODUCCIÓN}

La Naturaleza integra materiales, estructura, forma y función en un todo a través del proceso de crecimiento, que a su vez está influenciado por estímulos y limitaciones externas creadas por el medio ambiente. Las leyes inherentes a este proceso de crecimiento, crean una estructura jerárquica ascendente que permite la aparición de sistemas naturales optimizados que se adaptan y evolucionan para desempeñarse mejor en su entorno. Con un número limitado de elementos de la tabla periódica, la Naturaleza configura materiales de materia orgánica e inorgánica, integrando esta última en estructuras compuestas multifuncionales (Razghandi, 2014, p.3). Por lo tanto, los mismos "bloques de construcción" pueden crear una amplia gama de materiales naturales cambiando solo su configuración, generando soluciones robustas con un rendimiento máximo, utilizando recursos mínimos.

En comparación, las estrategias de fabricación hechas por los seres humanos, en su mayoría, se han basado en la extracción de materias primas naturales como recursos inagotables, convirtiéndose en un sistema insostenible. A través de una mayor conciencia de cuán limitados son estos recursos, está surgiendo un cambio de paradigma que nos lleva a cuestionarnos qué diseñamos, qué producimos y cómo lo hacemos, y una vez más, los diseñadores están recurriendo a la naturaleza y la biología para aprender de ella y crear soluciones bioinspiradas.

\section{SENTIR Y ACTUAR: ACTUADORES NATURALES.}

Las plantas poseen actuadores biológicos que les permiten sentir, reaccionar y adaptarse a los estímulos ambientales. Estos sistemas avanzados con funcionalidad integrada, pueden ser activos o pasivos; y crean una amplia gama de movimientos reversibles y cambios de forma en las plantas.

Los sistemas pasivos mantienen la capacidad de sentir y actuar incluso si están biológicamente muertos. Según Burgert y Fratzl (2009, p.1542) "Los sistemas pasivos se basan principalmente en tejidos ya muertos que están diseñados para sufrir cambios predeterminados ante cambios en las condiciones ambientales. Estos sistemas no requieren un mayor control o suministro de energía por parte del organismo una vez que se completa su crecimiento, lo que los hace bastante interesantes para la transferencia biomimética". Por lo tanto, las plantas con estos sistemas no necesitan realizar ningún proceso metabólico porque sus propiedades de actuación están embebidas morfológicamente dentro de la estructura del material.

Esta investigación se inspira en el mecanismo de actuación pasivo de la cápsula de semillas de la planta de hielo (Delosperma nakurense), que actúa en contacto directo con el agua, abriendo sus valvas al humedecerse para liberar las semillas. Las valvas de la planta de hielo están revestidas por tejidos especializados (quilla 
higroscópica), que en escala microscópica exhiben una estructura celular en forma de panal (Figura 1). Las partes huecas (lumen) de esta estructura anisotrópica están rellenas con material altamente dilatable que obliga a las células en forma de rombo a expandirse en una dirección preferencial tras la absorción de agua. En la escala macroscópica, dicha expansión unidireccional provoca el despliegue de las valvas, por lo que las quillas y la capa de respaldo pasiva de la valva funciona como una bisagra que permite grandes rotaciones (Razghandi et al., 2014).

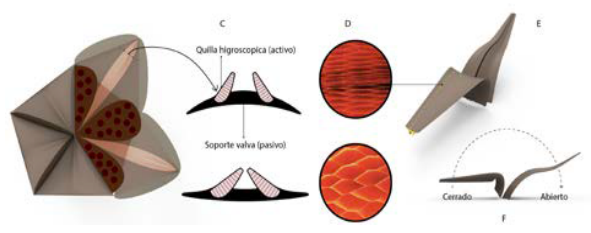

Figura 1 Esquema del mecanismo hidro-sensible de la planta de hielo. C. corte axial de la quilla higroscópica, D. corte transversal, representación de la expansión de la estructura celular, E y $F$ apertura y cierre. Adaptado de: (Razghandi et al., 2014)

Entre los distintos tipos de sistemas inspirados en plantas, las estructuras celulares presurizadas son las más estudiadas, debido a su potencial de transformación [...] Por lo general, consisten en una red de 'células' artificiales con geometría optimizada y propiedades elásticas; cuando algunas de estas celdas se presurizan y expanden (por ejemplo, mediante bombeo externo), la estructura general exhibirá cambios de forma y actuación prescritos (Li et.al, 2016).

\section{MATERIALES AUXÉTICOS}

Los auxéticos son una clase de metamateriales caracterizados por tener un módulo de Poisson negativo. El módulo de Poisson oscila entre -1.0 y +0.5 para los materiales isotrópicos, esto se refiere a materiales que responden de la misma manera en todas las direcciones tras una carga mecánica, independientemente de la dirección de la carga. Cuando se estira un material convencional, se contrae transversalmente a la dirección de la fuerza, se vuelven más delgados en su sección transversal y se expanden bajo compresión, un ejemplo típico de esto es el caucho. Los auxéticos, por otro lado, cuando se estiran, se expanden transversalmente a la fuerza aplicada, comportándose opuestamente a los materiales convencionales, tanto en extensión como en compresión, por lo tanto, tienen una relación de Poisson negativa. (Figura 2).
El diseño y la fabricación de estructuras auxéticas pueden exhibir una amplia gama de aplicaciones, además fabricarse en diferentes escalas y materiales base. Estos tipos de materiales tienen un gran potencial para diseñar y adaptar propiedades y comportamientos no convencionales usando la geometría como recurso principal.

\section{BIOINSPIRACIÓN}

Como ya se ha ampliado hasta cierto punto, los actuadores biológicos de las plantas muestran un alto potencial de transferencia biomimética. Este potencial se puede utilizar para crear materiales responsivos hechos por humanos, lo que da lugar a posibilidades sin precedentes para el diseño y la fabricación de materiales novedosos que interactúan con su entorno.

El propósito de la biomimética es la extracción, transferencia y aplicación del conocimiento adquirido a partir de modelos biológicos. Esta metodología se basa directamente en la capacidad del diseñador para establecer conexiones entre información que se encuentra en diferentes dominios. Una vez que se descubre el fenómeno biológico relevante, se puede aplicar un razonamiento analógico para transferir el conocimiento del dominio fuente (es decir, la biología) al dominio objetivo (es decir, el diseño).

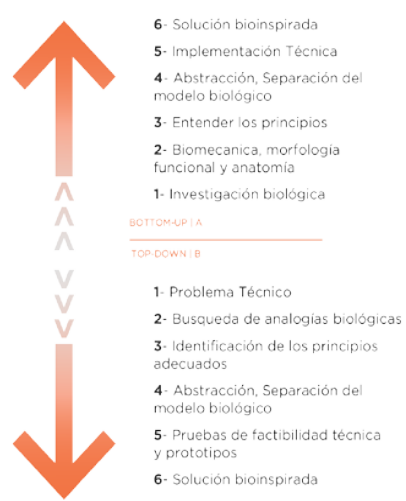

Figura 3 Proceso de investigación biomemetica A bottom-up B top Down

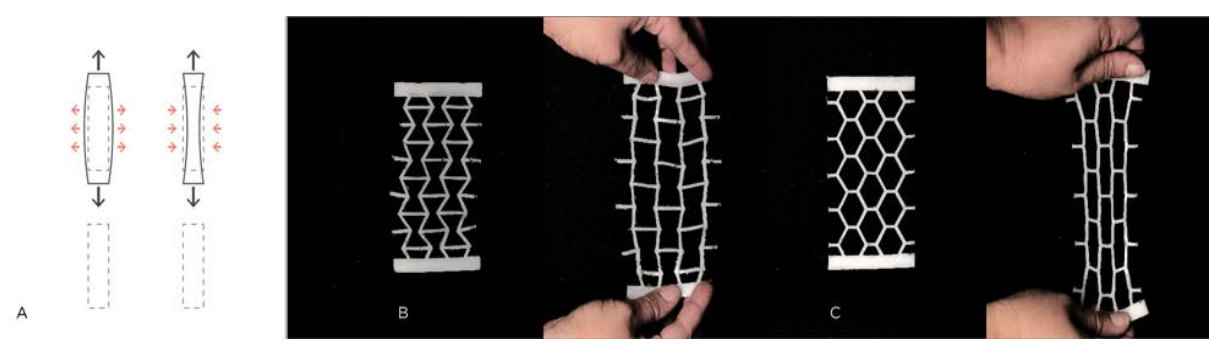

Figura 2 Estructura de panal entrante módulo de Poisson negativo= auxético (B) estructura de panal Módulo de Poisson positivo (c) -en ambos casos comportamiento bajo fuerza de tensión. 
La bioinspiración puede ocurrir ya sea por "empuje biológico", un fenómeno biológico que inspira una nueva aplicación (bottom-up) o por "tirón tecnológico", un problema técnico que busca resolverse observando cómo lo hace la naturaleza (top-down). La Figura 3 ilustra una versión sintetizada de ambos procesos. Imagen adaptada de Knippers y Speck, 2012).

A través de la ciencia, la comprensión de la Naturaleza con medios tecnológicos ha abierto el acceso a fenómenos biológicos que tienen el potencial de ser transferidos a campos como el diseño y la arquitectura. La transferencia biomimética del enfoque ascendente (bottom-up) de la Naturaleza da lugar al diseño de nuevos materiales y estructuras innovadoras, y abre oportunidades emocionantes para imaginar y crear aplicaciones que nunca antes se habían pensado. Uno puede interpretar las estrategias aprendidas de la naturaleza para crear tecnología aplicada.

Al emular la capacidad de la Naturaleza para estructurar la materia a través de estrategias morfológicas, la materia activa de diseñador también puede esforzarse por utilizar la cantidad mínima de recursos para un rendimiento máximo. En los resultados que se muestran en la próxima sección, la morfología se destaca como una de las estrategias principales a través de la cual los materiales convencionales pueden ser dotados de comportamientos no convencionales, con funcionalidades integradas que pueden detectar y actuar sobre diferentes estímulos.

\section{DISEÑO Y FABRICACIÓN DE MATERIALES MORFO-ACTIVOS}

En las últimas dos décadas, ha habido un aumento y expansión de los métodos de fabricación digital; no solo han avanzado, sino que también se han democratizado. Este aumento ha tenido un impacto en los diseñadores al permitir la participación directa en los procesos de fabricación que conducen a la aparición de una nueva "praxis material" en el diseño (Oxman, 2015). Hoy podemos ver un aumento de nuevos materiales cuyas propiedades y comportamientos pueden diseñarse en la estructura mesoscópica. "El papel crucial de la estructura mesoscópica distingue los "materiales de diseño" de las disciplinas más tradicionales como la ciencia de materiales y la química, que se enfoca en las escalas más pequeñas para manipular el orden de los bloques de construcción, como son los átomos y las moléculas ". (Reis et al., 2015, p.25-26).

En las secciones a continuación, los métodos de fabricación digital y la fabricación artesanal se han utilizado como una actividad de investigación experimental de creación y pensamiento.

Los modelos fabricados para esta investigación se llevaron a cabo en corte laser, usando como material de base foamy (goma EVA) de $3 \mathrm{~mm}$ de espesor. Se decidido usar este material y tecnología, ya que son altamente accesibles, está facilidad permitió hacer muchas pruebas en periodos cortos de tiempo.

El desarrollo del proyecto da como resultado el diseño y la fabricación de un material que se basa tanto en los actuadores de la planta de hielo como en los mecanismos de amplificación cinemáticos de las estructuras auxéticas. Una gama de materiales bioinspirados que cambian de forma, transformándose fuera del plano en respuesta a una fuerza aplicada, creando un material activo y sensible a través de la morfología.

Estos prototipos simples (Figura 4) funcionan como modelos mecánicos de un sistema de actuación que está morfológicamente embebido en la disposición espacial de las células auxéticas, que se caracterizan por tener un comportamiento de expansión contraintuitivo cuando se somete a una fuerza de tracción. Conceptualmente, estos prototipos se acercan a lo que se observa en la quilla higroscópica de la planta de hielo_que es la expansión de sus estructuras celulares que crea movimiento y transformación.

La inestabilidad mecánica / geométrica / elástica generalmente se asocia con fallas de materiales o colapso estructural a diferentes escalas de longitud; Algunos ejemplos son pandeo de estructuras, trituración de sólidos celulares, arrugas y grietas, crecimiento vacío, fractura y colapso. (Kochmann y Bertoldi, 2017). Los estudios y aplicaciones de ingeniería han tenido, durante mucho tiempo, el objetivo de prevenir este comportamiento estructural y material. Sin embargo, en las últimas décadas, se ha producido un cambio de paradigma, uno para diseñar, aprovechar y controlar las inestabilidades mecánicas en lugar de evitarlas. En la siguiente sección, el objetivo es abordar cómo los parámetros geométricos pueden influir en el pandeo y la flexión de las vigas y, por lo tanto, crear un comportamiento fuera del plano.

\section{CELDA UNITARIA}

Uno de los ejemplos más estudiados de geometría auxética es la celda de panal entrante presentada por Evans (Evans, 2000). En esta investigación, esta geometría se utiliza para desarrollar diferentes organizaciones. La razón para explorar este tipo de estructura es debido a su alta capacidad de deformación y su simplicidad geométrica. La deformación de esta estructura se logra principalmente mediante la flexión de las vigas entrantes y la compresión axial de las vigas horizontales.

Para la fabricación de estas estructuras celulares, hay cuatro parámetros a tomar en cuenta (Figura 4): la longitud de las vigas horizontales $B$, la longitud de las vigas reentrantes $\mathrm{L}$, el ángulo reentrante $\theta$, el espesor de la sección transversal de la viga E1 y el grosor E1 (lado izquierdo de la figura)

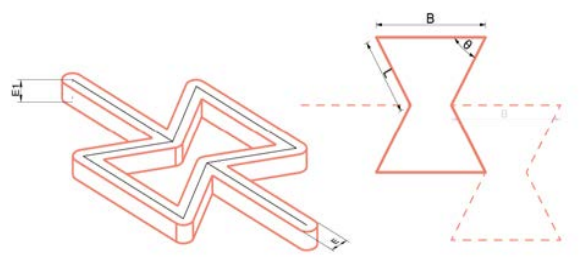

Figura 4 celda auxética de panal reentrante. Ilustración de los parámetros geométricos. 


\section{ESTUDIO DE MOVIMIENTO - LIBERTAD Y RESTRICCIÓN}

"Para cambiar drásticamente la forma de un objeto, debe poseer cierto grado de suavidad, ya sea en un sentido material, por ejemplo, tener un módulo elástico bajo, o en un sentido geométrico, por ejemplo, tener esbeltez". (Holmes, 2019, p.118)

Para llevar a cabo los estudios preliminares de movimiento se fabricaron unidades de estructura reentrante con un elemento restrictivo adicional utilizando (como metáfora) el criterio de estabilidad de Maxwell, ver: (Bathe et al.,2019). Esto permitió visualizar diferentes grados de rigidez y movimiento al someter las estructuras a una fuerza de tensión. (Figura 5).

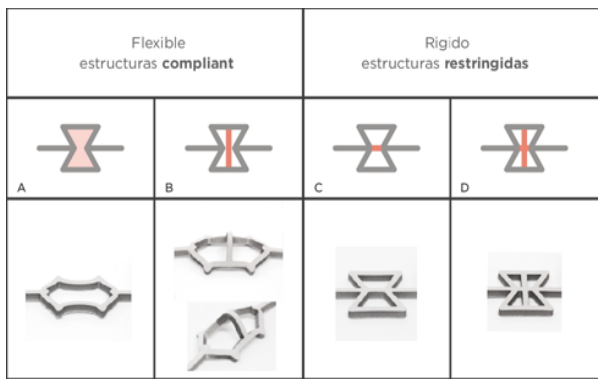

Figura 5 Tabla que muestra la relación entre la geometría y el movimiento en celdas de panal reentrantes auxéticas.

En las estructuras rígidas y restringidas (Figura 5), se observa que la viga alineada con la dirección de carga (Figura 5 C) y las vigas en cruz (Figura 5 D) crean el mismo efecto perceptible. Por lo tanto, para crear una celda rígida y restringida, usamos la viga alineada a la dirección de carga (B), en lugar de vigas en cruz (D) utilizando así menos material, a su vez logrando el mismo resultado.

\section{ORGANIZACIONES SIMPLES}

En el siguiente experimento, el objetivo era visualizar cómo una celda activada impacta a su "vecina". También se cambió la ubicación de los puntos de actuación en la estructura para lograr varios movimientos en una sola organización. (Figura 6 B3 y B4).

La transferencia de movimiento en estas estructuras se logra sin usar bisagras tradicionales, en este caso, la relación entre el material base y la geometría crea bisagras de flexión que se doblan y rotan, creando un mecanismo flexible (Compliant mechanism).

A través del diseño y la disposición espacial de estos elementos de viga elástica, bajo fuerza de tensión, no solo se doblan, sino que también se pandean. Generalmente, esto ocurre bajo compresión, y en algunos casos como este (Figura 6 B 1-5), las inestabilidades geométricas ocurren bajo tensión permitiendo grandes deformaciones. Este es el caso del elemento de viga transversal que está bajo compresión cuando la celda está bajo tensión. Con el estudio mostrado anteriormente, se puede observar intuitivamente cómo un pequeño cambio local en la geometría y / o en la disposición espacial puede conducir a un gran cambio en la transformación tanto plana como fuera del plano.

En la figura 7 se puede observar que, al poner la línea de la viga transversal contra una superficie, la tira B4 de la figura 4 , crea una transformación casi total fuera del plano. La tira desarrollada en esta investigación se transforma a través de la combinación de la forma y el aprovechamiento de las inestabilidades geométricas (pandeo)

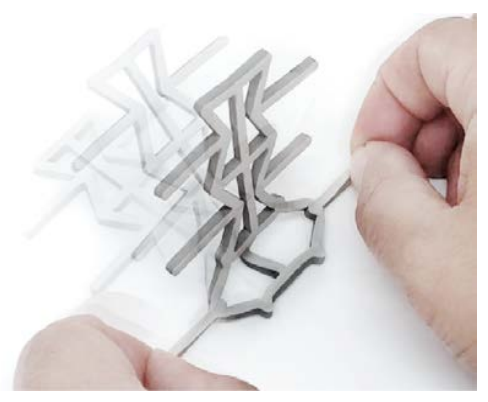

Figura 7 tira con transformación vertical fuera del plano.

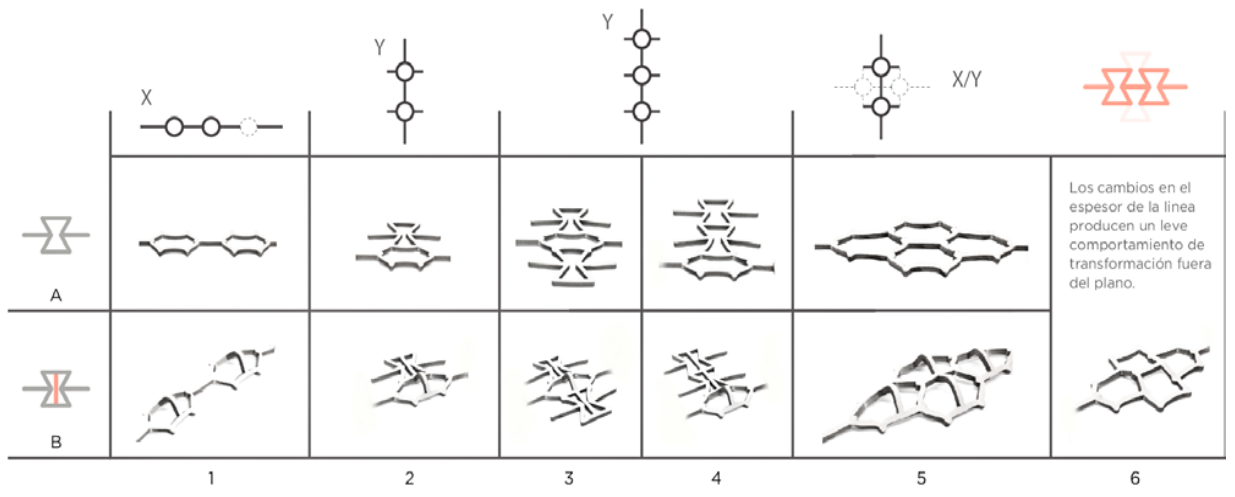

Figura 6 Tabla que muestra la relación entre la geometría y el movimiento dentro de las celdas vecinas en estructuras simples de nido de abeja reentrantes auxéticas // extracción desde el punto de actuación en línea 


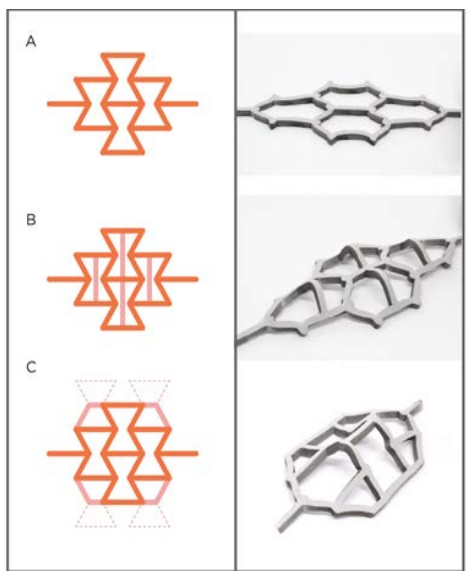

Figura 8 Diseño de comportamientos complejos a partir de restricciones geométricas simples que crean inestabilidades materiales

\section{DOMOS Y PLIEGES}

Cada organización es una estructura en sí misma, con un comportamiento distintivo, derivado no solo de la suma de las partes sino a través de una disposición espacial atentamente planeada. En la combinación de una región restringida y una flexible, se pueden crear estructuras más complejas para aprovechar inestabilidades geométricas que permitan lograr la transformación deseada fuera del plano.

El aporte de todas las exploraciones anteriores permitió comprender cómo la disposición del material y la geometría puede afectar la forma en que un modelo determinado se transforma tanto local como globalmente. Este input informó cómo crear celdas con deformación dirigida fuera del plano (región activa) y deformación en el plano (región pasiva, restringida) Figura 9, el objetivo de final de esta investigación es combinarlas y crear estructuras que muestren comportamientos más complejos.

Mientras que en la estructura celular de la planta de hielo, el material hidroabsorbente se hincha y como resultado las células se expanden y las valvas se abren, en los "modelos" (Figura 9) la fuerza de tracción expande las células auxéticas, y debido a la disposición espacial diseñada, se crea el movimiento (flexión y / o pandeo), que obliga a toda la estructura a transformarse fuera del plano, convirtiendo las estructuras $2 \mathrm{~d}$ en superficies espaciales $3 \mathrm{~d}$ y / o mecanismos de apertura y cierre.
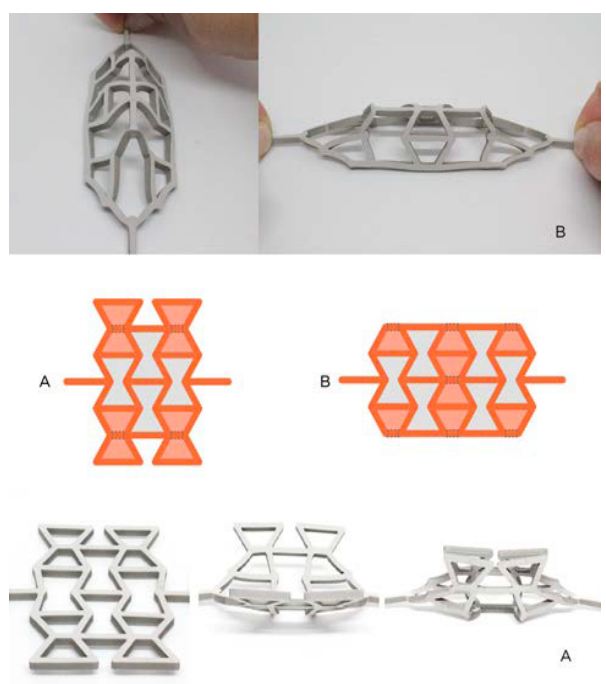

Figura 9 Diferentes organizaciones que generan transformaciones globales fuera del plano creando estructuras tipo domo (B) y sistemas de cierre (A)

\section{GRIPPER}

Este último modelo demuestra cómo se puede embeber una respuesta específica, diseñando la geometría y la disposición espacial de una estructura y de esa manera crear funcionalidades avanzadas.

Esta nueva funcionalidad se realizó mediante el uso de la misma estructura que la que se muestra en la Figura 9 (abajo). al unir asignar dos puntos de activación y tirar desde el medio, se obtiene una transformación global más compleja como se muestra a continuación.

En la Figura 10 se puede ver como este material laminar al ser sometido a una fuerza de tensión, cambia su morfología global y es capaz de agarrar, contener y levantar una pelota de ping-pong. Este nuevo comportamiento motiva de inmediato las posibles aplicaciones en el campo de la robótica blanda. (Soft robotics)
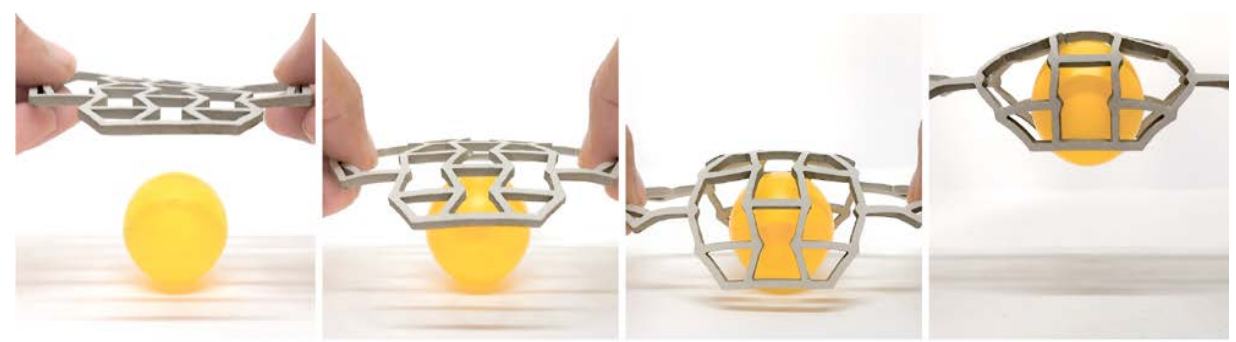

Figura 10 Gripper \# 1 recogiendo una pelota de ping-pong 


\section{CONCLUSIÓN}

"El diseño está formado por fuertes tensiones, como han escrito Sabine Ammon y Eva-Maria Froschauer, porque 'fluctúa entre tirar la línea y sobrepasarla constantemente, encarna tanto la vaguedad como los imperativos de precisión y explicación, y se desarrolla a partir de la inspiración repentina mientras exige una elaboración sistemática' ". (Mareis, 2017, p.73)

Uno de los principales desafíos de esta investigación fue crear una interpretación y una abstracción que permitieran descomponer el complejo mecanismo natural que se observa en la quilla higroscópica de la planta de hielo en ideas más simples. Permitiendo la transferencia al dominio del diseño para la creación de un modelo aplicado que de cuenta de ese sistema pasivo.

El otro desafío fue determinar una forma simple y accesible de fabricar estructuras complejas de transformación formal a partir de materiales planos. Los metamateriales mecánicos auxéticos, al permitir cambiar el comportamiento en el plano de las células individuales y combinarlos de varias maneras, como se ha demostrado, nos permitieron explorar la transformación fuera del plano embebida en el material base.

Creo que el aspecto más emocionante de esta investigación es proponer el uso de la geometría como una forma de prescribir o programar el comportamiento de un material. Se ha demostrado cómo agregando un "bloqueo" geométrico a las células individuales puede influir en la respuesta mecánica de toda la estructura. En el material diseñado en esta investigación, no hay partes o mecanismos discretos para lograr movimiento y transformación; en cambio, hay regiones activas y restringidas en una lámina flexible, que cuando se somete a tensión uniaxial, se dobla y se pandea para formar estructuras tridimensionales y movimientos fuera del plano. Este comportamiento de transformación tridimensional provocó de inmediato algunas aplicaciones en el campo de la robótica blanda (soft robotics) y una nueva estrategia potencial para pinzas blandas (Grippers) de accionamiento basado en geometría.

Las exploraciones materiales demostraron ser una parte fundamental del proceso. Solo con ese tipo de exploraciones que pude llegar a comprender la funcionalidad de las estructuras y cómo se pueden usar. A través de esta investigación interdisciplinaria, fue posible tejer conceptos de biología, ciencia de materiales e ingeniería desde la perspectiva de una diseñadora. Esta forma de abordar el diseño y fabricación de materiales promueve prácticas artesanales y proyectuales para incorporar a la investigación científica, utilizando la producción digital y práctica para crear nuevos "materiales activos".

Con los resultados se logra la integración total: forma, material y comportamiento, todo en uno. Este articulo da cuenta de la iteración actual de esta investigación y ha abierto una gran cantidad de posibilidades para futuras exploraciones.

\section{REFERENCIAS}

Bhate, D., Penick, C., Ferry, L., \& Lee, C. (2019). Classification and Selection of Cellular Materials in Mechanical Design: Engineering and Biomimetic Approaches. Designs, 3(1), 19. https://doi.org/10.3390/designs3010019

Burgert, I. and Fratzl, P. (2009) 'Actuation systems in plants as prototypes for bioinspired devices', Philos Trans A Math Phys Eng Sci, 367(1893), pp. 1541-57.

Cho, H., Seo, D., \& Kim, D.-N. (2019). Mechanics of Auxetic Materials. In Handbook of Mechanics of Materials (pp. 733757). Springer Singapore. https://doi.org/10.1007/978-981-106884-3_25

Evans, K. E., \& Alderson, A. (2000). Auxetic materials: Functional materials and structures from lateral thinking! Advanced Materials, 12(9), 617-628. https://doi.org/10.1002/(SICI)15214095(200005)12:9<617::AID-ADMA617>3.0.CO;2-3

Holmes, D. P. (2019, April 1). Elasticity and stability of shapeshifting structures. Current Opinion in Colloid and Interface Science. Elsevier https://doi.org/10.1016/j.cocis.2019.02.008

Knippers, J., \& Speck, T. (2012). Design and construction principles in nature and architecture. Bioinspiration and Biomimetics, $7(1)$. https://doi.org/10.1088/17483182/7/1/015002

Kochmann, D. M., \& Bertoldi, K. (2017, September 1). Exploiting Microstructural Instabilities in Solids and Structures: From Metamaterials to Structural Transitions. Applied Mechanics Reviews. American Society of Mechanical Engineers (ASME). https://doi.org/10.1115/1.4037966

Li, S., \& Wang, K. W. (2016). Plant-inspired adaptive structures and materials for morphing and actuation: a review. Bioinspiration \& Biomimetics, 12(1), 011001. https://doi.org/10.1088/1748-3190/12/1/011001

Mareis, C (2017). "Designerly Ways of Knowing."The Impertinent Promise of an Epistemic Culture of Design . In: Doll, N., Bredekamp, H., Schäffner, W., Exzellenzcluster "Bild Wissen Gestaltung. Ein interdisziplinäres Labor", \& Martin-GropiusBau. (2017). +ultra knowledge \& gestaltung., Berlin. Leipzig: E. A. Seemann, S. 71-77.

Oxman, Rivka (August 17, 2015) MFD: Material-FabricationDesign: A Classification of Models from Prototyping to Design. Proceedings of the International Association for Shell and Spatial Structures (IASS)

Razghandi, K., Bertinetti, L., Guiducci, L., Dunlop, J. W. C., Fratzl, P., Neinhuis, C., \& Burgert, I. (2014). Hydro-actuation of ice plant seed capsules powered by water uptake. Bioinspired, Biomimetic and Nanobiomaterials, 3(3), 169-182. https://doi.org/10.1680/bbn.14.00016

Reis, P. M., Jaeger, H. M., \& van Hecke, M. (2015). Designer Matter: A perspective. Extreme Mechanics Letters, 5, 25-29. https://doi.org/10.1016/j.eml.2015.09.004 